Louisiana State University

LSU Digital Commons

$1-1-2015$

\title{
Photoactivated miR-148b-nanoparticle conjugates improve closure of critical size mouse calvarial defects
}

\author{
Ammar T. Qureshi \\ Louisiana State University \\ Andrew Doyle \\ Louisiana State University \\ Cong Chen \\ Louisiana State University \\ Diana Coulon \\ LSU Agricultural Center \\ Vinod Dasa \\ LSU Health Sciences Center - New Orleans
}

See next page for additional authors

Follow this and additional works at: https://digitalcommons.Isu.edu/animalsciences_pubs

\section{Recommended Citation}

Qureshi, A., Doyle, A., Chen, C., Coulon, D., Dasa, V., DelPiero, F., Levi, B., Monroe, W., Gimble, J., \& Hayes, D. (2015). Photoactivated miR-148b-nanoparticle conjugates improve closure of critical size mouse calvarial defects. Acta Biomaterialia, 12 (1), 166-173. https://doi.org/10.1016/j.actbio.2014.10.010

This Article is brought to you for free and open access by the School of Animal Sciences at LSU Digital Commons. It has been accepted for inclusion in Faculty Publications by an authorized administrator of LSU Digital Commons.

For more information, please contact ir@lsu.edu. 
Authors

Ammar T. Qureshi, Andrew Doyle, Cong Chen, Diana Coulon, Vinod Dasa, Fabio DelPiero, Benjamin Levi, W. Todd Monroe, Jeffrey M. Gimble, and Daniel J. Hayes 


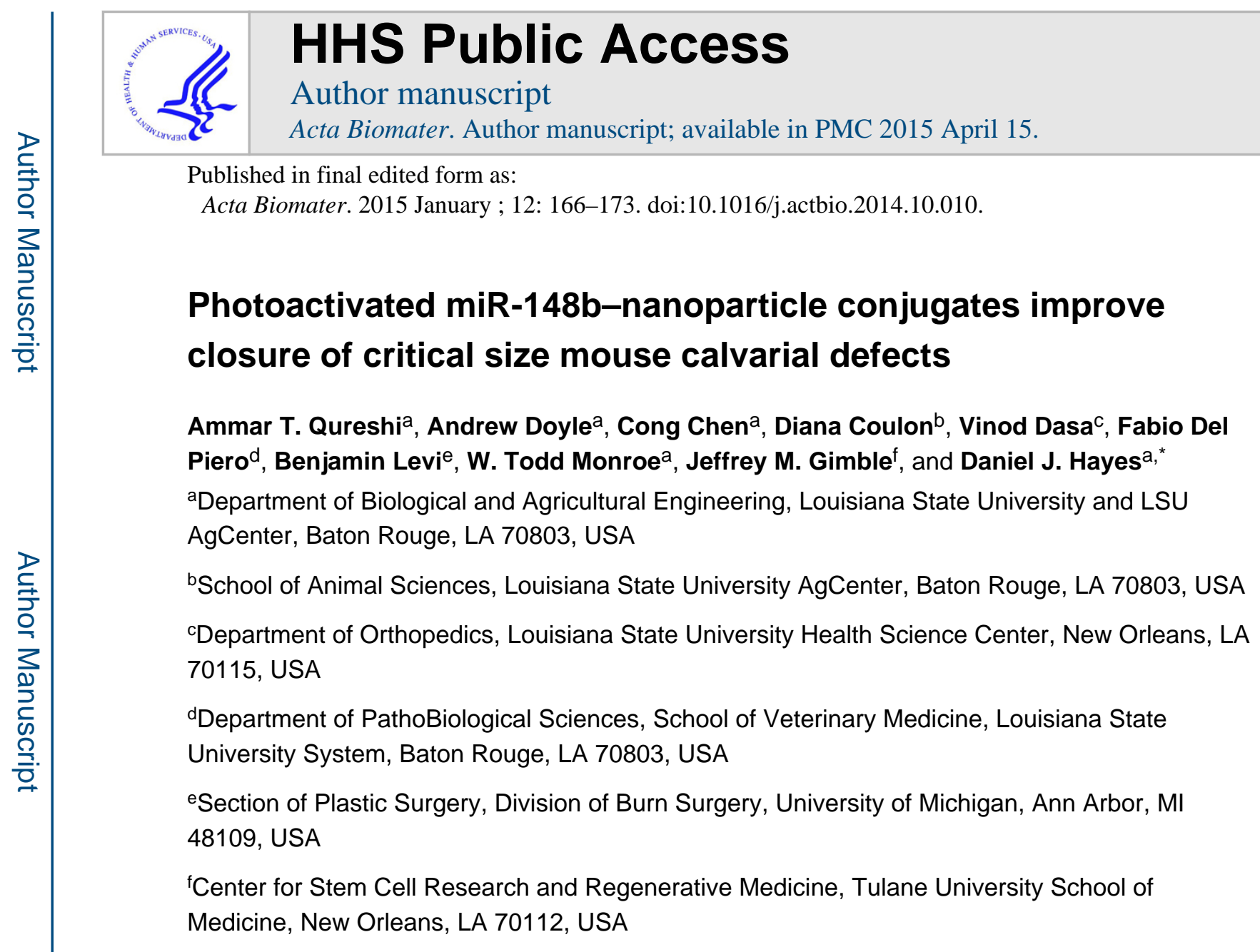

\section{Abstract}

Inducible systems providing temporal control of differentiation have the potential to improve outcomes in surgical reconstruction and regenerative medicine by precise modulation of wound healing and tissue repair processes. The aim of this study was to demonstrate that nanoformulated microRNA (miRNA) conjugates activated via photo exposure can lead to the induced osteogenic differentiation of human adipose-derived stromal/stem cells (hASCs) in vivo. The conjugate PCmiR-148b-SNP, a mimic of miRNA-148b tethered to silver nanoparticles (SNPs) via a photolabile linker, was used to modulate gene expression for improved closure of a critical size defect drilled on the right parietal bone of male CD-1 nude homozygous mice. The PC-miR-148b-SNP conjugates added to hASCs and loaded to either Matrigel or polycaprolactone (PCL) scaffolds resulted in different levels of healing of the defect. After 4 and 12 weeks, 3-D micro-computed tomography reconstructed images indicate statistically significant defect closure from $3.83 \pm$ $1.19 \%$ to $5.46 \pm 2.01 \%$ and $6.54 \pm 4.28 \%$ to $32.53 \pm 8.3 \%$ for non-photoactivated and photoactivated conjugates, respectively, in the PCL scaffolds. The results were confirmed with H\&E and Masson's Trichrome stains in the transverse sections of photoactivated conjugates. Collagen fiber staining was greatest at 12 weeks when it reached approximately the same density

(C) 2014 Acta Materialia Inc. Published by Elsevier Ltd. All rights reserved.

*Corresponding author. Tel.: +1 (225) 578 2919; fax: +1 (225) 578 3492. danielhayes@lsu.edu (D.J. Hayes).

Disclosures

J.M.G. is a co-owner, co-founder and Chief Scientific Officer at LaCell LLC, a biotechnology company focusing on the use of stromal/stem cells for regenerative medical therapies. 
and thickness as the native calvarium. This technology provides a platform that can be used with other miRNAs that actively govern the pathways responsible for regenerative and wound healing processes.

\section{Keywords}

Calvarial size defect (CSD); Osteogenic differentiation; Photoactivated delivery system; miR-148b

\section{Introduction}

Currently, allogeneic or autogenic graft materials are widely used for the repair of skeletal bone defects but these techniques have disadvantages including rejection, infection, limited supply and post-operative donor site morbidity [1]. Adipose tissue is an abundant and accessible source of stromal/stem cells that is isolated commonly from patients through body contouring and liposuction surgeries [2]. The derived multipotent human adiposederived stromal/stem cells (hASCs) have the potential to differentiate along the adipocyte, chondrocyte, myocyte, neuronal, and osteoblast lineages [3,4]. These hASCs have shown utility in the repair and regeneration of acute and chronically damaged tissues if activated with appropriate inductive factors $[5,6]$.

While the majority of the established differentiation factors are proteins, microRNAs (miRNA) —non-coding RNAs involved in post-transcriptional regulation pathways—are increasingly being explored for their potential in modulating the osteogenic differentiation of hASCs [7-9]. miRNAs play similar roles to protein-based growth factors in that they can act on hundreds of targets and miRNAs are now known to regulate cellular processes and fate transitions. Relatively few miRNAs, namely miR-26a, $-148 b$, -27 a and -489 , have been shown to regulate osteogenesis in mesenchymal stem cells (MSCs) $[7,9,10]$. Of these compounds, miR-148b has been shown to induce de novo osteogenesis as indicated by increases in total alkaline phosphatase activity and early specific biomarker expression [7]. To demonstrate a more targeted form of exogenous miRNA activity for directed differentiation, our group recently described a miR-148b-modulated photoactivated miRNA delivery system with the capability to upregulate alkaline phosphatase (ALP) and osteocalcin $(\mathrm{OCN})$ and mineralization of hASCs in vitro [11]. The miRNA-mediated technique could serve as an attractive therapeutic modality for regenerative medicine and is of particular interest in the design of therapies for critical size defects (CSDs), spinal fusion and skeletal reconstruction.

Several reports have described the therapeutic advantages of delivering stem cells with growth and inductive factors within biodegradable scaffolds that provide appropriate physiological environments [12-14]. CSD models have been used for both the evaluation of normal healing, and for hASC-mediated closure [15]. Here we have adapted a previously described mouse CSD model $[16,17]$ for the proof-of-concept evaluation of photomodulated repair by hASCs transfected with photoactivated miRNA-148b-silver nanoparticle (SNP) conjugates. hASCs were loaded onto thermally precipitated polycaprolactone (PCL) scaffolds or into Matrigel ${ }^{\mathrm{TM}}$ thermoreversible hydrogel before being 
treated with miRNA-148b-SNP conjugates. Soon after, conjugates were photoactivated and hASC-scaffold or hASC-gel composites were surgically implanted in defect sites for up to 12 weeks. The in vivo osteogenic capability of hASCs was evaluated histologically and defect closure was assessed via micro-computed tomography ( $\mu \mathrm{CT})$ analysis [17].

This photoactivated miRNA delivery system provides a greater temporal control of differentiation in vivo and may potentially lead to improved healing of CSDs. It has very little "leak" or uninduced activity, hence hASCs transfected with photoactivated-miR-148b delivery conjugates remain inert until photo-exposed at the appropriate wavelength and dosage. Additionally, since intracellular miRNA are short lived and only transiently modulate mRNA expression, it is expected that there is little chance of permanent activity once an activated cell has begun differentiation. Thus, our photoactivated miRNA delivery system can enhance hASC osteogenic differentiation and demonstrates significant translational potential.

\section{Materials and methods}

\subsection{Dosage dependence mineralization}

Varying concentrations of PC-miR-148b-SNP conjugates (10-100 $\mu$ of $227.2 \pm 1.7 \mathrm{ppm}$ SNP or $0.120-1.20 \mathrm{nM}$ ) were added to hASCs and ALP upregulation and mineralization of hASCs were tested at day 7 and 14, respectively. ALP upregulation was qualitatively and quantitatively measured by Millipore kits SCR 004 and SCR 066, while mineralization was quantified with Alizarin Red S. The calcium deposition and ALP absorbance expressed by the optical density of the aliquots were measured with a plate reader and normalized to the number of hASCs, measured by PicoGreen (Life Technologies, Eugene, OR).

\subsection{Ex vivo mineralization of PCL scaffolds}

To confirm the in vivo mineralization of PCL scaffolds with PC-miR-148b-SNP in mice, scaffolds were prepared as previously described Qureshi et al. [18]. Scaffolds were submerged in stromal media (SM; Dulbecco's Modified Eagle's Medium (DMEM), 10\% fetal bovine serum (FBS) and 1\% antibiotic) for 4 weeks while exchanging the media every 3-4 days. The mineralization was quantified with Alizarin Red S as previously described by Qureshi et al. [11].

\subsection{Osteogenic comparison of $\mathrm{PCL}$ and Matrige $\mathrm{I}^{\mathrm{TM}}$ in vitro}

hASCs were seeded in either $100 \mu$ l of Matrigel ${ }^{\mathrm{TM}}$ or PCL scaffolds for 3 weeks in vitro to compare the osteogenic effects of each material and evaluate the utility of each material to serve in conjunction with the PC-miR-148b-SNP + UV system. At the end of 3 weeks, Alizarin Red S was used to quantify extracellular mineralization. Samples included two negative control groups cultured in stromal media (hASCs + Matrigel ${ }^{\mathrm{TM}}$ and hASCs + PCL), two positive control groups cultured in osteogenic media (hASCs + Matrigel $^{\mathrm{TM}}$ and hASCs + PCL), and four experimental groups cultured in stromal media (PC-miR-148b-SNP + PCL, PC-miR-148b-SNP + UV + PCL, PC-miR-148b-SNP + Matrigel $^{\mathrm{TM}}$, and PC-miR-148b$\mathrm{SNP}+\mathrm{UV}+$ Matrigel $^{\mathrm{TM}}$ ). 


\subsection{Ethics and animal use}

hASCs were harvested and enriched from human lipoaspirate as previously described [5]. All tissues were obtained with informed consent under a clinical protocol reviewed and approved by the Institutional Review Board at the Pennington Biomedical Research Center. All animal housing conforms to NIH Guide standards and the Animal Welfare Act.

In vivo studies were conducted on adult (42 day old) male CD-1 nude homozygous mice 20-40 g (Jackson Laboratory J: NU Stock No. 007850). The Animal Facility was only accessed by authorized personnel who followed personal protective equipment (PPE) protocols. The mice were housed in autoclaved cages; given sterile food and water; and kept in barrier conditions, ambient temperature, 30-70\% humidity, and a $12 \mathrm{~h}$ light-dark cycle. This animal study was approved by LSU IACUC, protocol No. 13-004.

\subsection{Calvarial size mouse defects}

To avoid incompatibility of xenografted human tissue, athymic mice were used as model organisms [19]. Adult male CD-1 nude mice were anesthetized with inhalant isoflurane before a 1-1.5 cm skin incision was made on the sagittal midline to expose the right parietal bone. Non-healing, critical-sized calvarial defects $(4 \mathrm{~mm})$ were created in the right parietal bone using a 4.6 OD Trephine Bur (ACE Surgical, Brockton, MA) and a Dentalaire Prestige Dental Station (DTP00511, Fountain Valley, CA), while the dura mater was left undisturbed. The hASC-loaded scaffolds and gel with PC-miR-148b were then implanted in the defect. The surgical sites were sealed with Michel clips and tissue glue and the animals were monitored according to established post-operative animal care protocols. At either 4 or 12 weeks, the animals were killed by $\mathrm{CO}_{2}$ asphyxiation and cervical dislocation to analyze the effect of photoactivated miR-148b-SNP conjugates on calvarial defect closure.

\subsection{Calvarial size defect surgery preparation}

hASCs were seeded onto either pure PCL (4 mm diameter and $1 \mathrm{~mm}$ thick) scaffolds, as previously described by Qureshi et al. [18], or $100 \mu$ of Matrigel $^{\mathrm{TM}}$. Roughly $1.5 \times 10^{5}$ cells were suspended in $200 \mu \mathrm{l}$ of stromal media and seeded onto PCL scaffolds and thermoresponsive Matrigel ${ }^{\mathrm{TM}}$ by pipetting $10 \mu \mathrm{l}$ of cell suspension solution on each side of the scaffold and $20 \mu \mathrm{l}$ into the gel. Upon cell seeding, scaffolds were incubated at $5 \% \mathrm{CO}_{2}$ and $37^{\circ} \mathrm{C}$ for $2 \mathrm{~h}$ before submerging in $300 \mu \mathrm{l}$ of stromal media for $24 \mathrm{~h}$ prior to PCmiR-148b-SNP construct treatment. $50 \mu$ of PC-miR148b-SNP construct $(0.60 \mathrm{nM})$ was added to the scaffolds and gel for $16 \mathrm{~h}$ before exposure to light at $365 \mathrm{nM}$ for $5 \mathrm{~min}(52.4 \mathrm{~J}$ $\mathrm{cm}^{-2}$ ) by means of a GreenSpot photocuring system [20] (see Graphical abstract).

Animals were split into four experimental groups, two positive and four negative controls, and were tested for closure of the defect at 4 and 12 weeks. The experimental groups included (1) PC-miR-148b-SNP + PCL, (2) PC-miR-148b-SNP + Flashed + PCL, (3) PCmiR-148b-SNP + Matrigel $^{\mathrm{TM}}$ and (4) PC-miR-148b-SNP + Flashed + Matrigel $^{\mathrm{TM}}$. Negative controls included (1) PCL scaffold only, (2) Matrigel ${ }^{\mathrm{TM}}$ only, (3) empty defect only and (4) PCL scaffold with miSpike-21mer (RNA internal control). The positive controls included (1) PCL scaffold with hASCs and (2) PCL scaffolds with hASCs incubated in osteogenic media (OM; DMEM, 10\% FBS, $0.1 \mu \mathrm{M}$ dexamethasone, $50 \mu \mathrm{M}$ ascorbate-2-phosphate, 10 
$\mathrm{mM} \beta$-glycerophosphate and $1 \%$ antibiotic) for 7 days prior to implantation ( $n=3$ each). Prior to implantation, cell-seeded scaffolds were rinsed in sterile PBS to prevent transfer of medium-derived growth factors. After implantation, animals were allowed to recover under heating pads for $1 \mathrm{~h}$ before being returned to the animal facility. Thereafter, animals were observed once daily for 3 days and weekly thereafter to ensure postoperative recovery.

\subsection{In vivo imaging}

$\mu \mathrm{CT}$ was performed using a SkyScan 1074 tomography imaging system (Micro Photonics, Allentown, PA, USA) with the following settings: $40 \mathrm{kVp}$ polychromatic X-ray beam; field of view: $28 \mathrm{~mm}$ in diameter and $19 \mathrm{~mm}$ in height; current: $1000 \mu \mathrm{A}$; and exposure time: 420 $\mathrm{ms}$ for each of the 360 rotational steps. The 2-D projection images were used to reconstruct tomograms with a commercial package (Avizo 8, Mercury Computer Systems, Berlin, Germany). For each mouse, the percentage closure of the defect was normalized to the undamaged skull in Image J (NIH, Bethesda, MD) [16].

\subsection{Histological staining}

Following radiographic imaging, calvaria were processed and stained as described previously [15,16]. Briefly, the calvaria were harvested, fixed in $10 \%$ formalin for $24 \mathrm{~h}$, decalcified with $10 \%$ ethylenediaminetetraacetic acid (EDTA) for 3 days and embedded in paraffin wax for sectioning. To evaluate bone regeneration, $10 \mu \mathrm{m}$ sections were cut and stained with H\&E and Masson's Trichrome (American MasterTech, Lodi, CA, Item No. KTTRBPT) according to the manufacturer's protocols and imaged under bright-field illumination with an Olympus BX46 microscope at $10 \times$ magnification, where collagen appears pink and blue under H\&E and Masson's Trichrome stains, respectively.

\subsection{In vivo quantification of silver distribution}

After 12 weeks, the mice were killed and the liver, kidneys, spleen and brain were harvested, fixed in 10\% formalin for $24 \mathrm{~h}$ and analyzed for SNP concentration with inductively coupled plasma optical emission spectroscopy (ICP-OES). A $0.1 \mathrm{mg}$ sample of each organ was digested with piranha etch solution (3:1 mixture of sulfuric acid and hydrogen peroxide), transferred to weighed ICP vials, agitated for $2.5 \mathrm{~h}$ and diluted to a final volume of $10 \mathrm{ml}$ in nitric acid acidified water. The final solutions were weighed and diluted samples were analyzed via Varian Vista MPX (Palo Alto, CA).

\subsection{Statistical analysis}

All results were expressed as mean \pm standard error. Two-way ANOVA was used to measure the closure of the CSD while the other results were analyzed with a one-way $t$-test. For all comparisons, a $P$-value $<0.05$ was considered significant.

\section{Results}

\subsection{Osteogenic differentiation with varying concentrations of PC-miR-148b-SNP conjugates}

Prior to the implantation of scaffolds with PC-miR-148b-SNP conjugates in mice, the optimal concentration of conjugates to maximize the differentiation of hASCs was tested in 
vitro by exposing varying concentrations of conjugates to cells for 7 and 14 days and assessing ALP expression and Alizarin Red staining.

ALP is one of the early enzymes expressed during osteogenesis and is displayed on the extracellular portion of the plasma membrane, resulting in increased local concentrations of inorganic phosphate. The negative controls, hASCs cultured in stromal media (SM) and SM + Flashed, exhibited minimal ALP activity (Fig. 1A). The most significantly different ALP results were seen between the $0.12 \mathrm{nM}$ photoactivated and non-photoactivated conjugate. The normalized absorbance of ALP stain to the number of hASCs increased from $7.01 \times$ $10^{-5} \pm 1.44 \times 10^{-5}$ to $2.3 \times 10^{-4} \pm 5.75 \times 10^{-5}$ when photoactivated $(P<0.05$; one-sample $t$-test). The ALP absorbance values of photoactivated 0.60 and $1.20 \mathrm{nM}$ of conjugate samples were not significantly different from each other $(P>0.05$; one-sample $t$-test $)$ and likely lower than photoactivated $0.12 \mathrm{nM}$ conjugate sample due to fewer cells remaining in these samples. Concentrations above $0.60 \mathrm{nM}$ appear to impact cell number, potentially as a result of increased cytotoxicity, as indicated by a decrease in cell count after 7 days (Fig. 1B).

A late stage indicator of bone formation is the accretion of extracellular calcium deposits on extracellular matrix (ECM) deposited by the osteoblasts. For this dosage dependence study, the calcium accretion was quantitatively and qualitatively measured by Alizarin Red S stain as hASCs were treated with the same doses of conjugates used in previous assays (Fig. 2). The negative controls, hASCs cultured in stromal media (SM) and SM + Flashed, had the least calcium deposition (Fig. 2A and E), whereas the calcification of hASCs increased sharply for the photoactivated 0.12 and $0.60 \mathrm{nM}$ conjugate samples as the normalized absorbance increased to $9.88 \times 10^{-6} \pm 1.26 \times 10^{-6}$ and $1.03 \times 10^{-5} \pm 4.3 \times 10^{-7}$, respectively (Fig. 2F, G and I). The $1.20 \mathrm{nM}$ conjugate sample had the lowest number of hASCs and resulted in a lower normalized absorbance/hASC ratio. The morphological difference between cells transfected with lower $(0.12 \mathrm{nM})$ and higher $(1.20 \mathrm{nM})$ doses of PC-miR-148b-SNP conjugates can be visualized in Fig. 2F-H. At the highest concentration, the cells lose their "fibroblast-like" morphology and their membranes appear compromised, with substantial amounts of cellular debris present.

\subsection{Ex vivo mineralization of hASC-loaded PCL scaffolds}

The ex vivo mineralization of PCL scaffolds loaded with hASCs and conjugates was quantified with Alizarin Red S after 4 weeks (Fig. 3). During mineralization, osteoblasts produce extracellular calcium deposits, which are an indication of bone formation. The negative controls (scaffolds with hASCs and scaffolds with miSpike-21mer) induced minimal mineralization of the scaffolds, but the positive control, hASCs differentiated in $\mathrm{OM}$ prior to implantation, showed a significantly higher mineralization $(0.21 \pm 0.008)$. The difference in the normalized mineralization values between the non-photoactivated 0.12 and $0.60 \mathrm{nM}$ conjugate samples are not significantly different from each other. Even in samples treated with conjugates at higher concentrations and not photoactivated, the conjugate remained inert and did not result in the mineralization of hASCs in the scaffolds, indicating no "leak" in the uninduced state. For the photoactivated samples, the normalized mineralization of scaffolds increased to $1.09 \times 10^{-5} \pm 6.03 \times 10^{-5}$ and $1.34 \times 10^{-5} \pm 6.43 \times$ 
$10^{-7}$ for 0.60 and $0.12 \mathrm{nM}$ samples, respectively, showing statistical difference from their respective non-photoactivated controls $(P<0.05$; one-sample $t$-test). Based on these results of this ex vivo experiment $0.60 \mathrm{nM}$ was chosen for subsequent in vivo CSD closure studies.

\subsection{Osteogenic comparison of $\mathrm{PCL}$ and Matrige $\mathrm{T}^{\mathrm{TM}}$ in vitro}

The in vitro mineralization of PCL scaffolds and Matrigel ${ }^{\mathrm{TM}}$ loaded with hASCs and treated with the PC-miR-148b-SNP system was evaluated with Alizarin Red S after 21 days (Fig. 4). During mineralization, osteoblasts produce extracellular calcium deposits, which are an indication of bone formation. Matrigel ${ }^{\mathrm{TM}}$ had a greater overall osteoinductive effect on hASCs than PCL. The mineralization of those cells treated with photoactivated PCmiR-148b-SNP in Matrigel ${ }^{\mathrm{TM}}(0.240 \pm 0.02)$ displayed a significantly higher absorbance than those photoactivated in PCL scaffolds $(0.196 \pm 0.02 ; P<0.05$; one-sample $t$-test) (Fig. 4).

\section{4. $\mu \mathrm{CT}$ imaging}

2-D and 3-D $\mu \mathrm{CT}$ image reconstruction of mice skulls were taken after 4 and 12 weeks for the experimental and test control groups to assess CSD closure. Defect closure in the negative controls was minimal at 12 weeks post implantation; corresponding to closure values of $5.97 \%, 4.76 \%$ and $1.52 \%$ for PCL scaffold only, empty defect and PCL scaffold + miSpike-21mer, respectively (Fig. 5A and B). PCL scaffolds loaded with hASCs and maintained in stromal media showed moderate bone regrowth. For the PCL scaffold + hASC sample, the percentage closure of the defect was not significantly different between 4 and 12 weeks as the values changed from $4.47 \pm 3.65 \%$ to $4.6 \pm 1.98 \%$. Conversely, the preimplantation osteogenic induction of the scaffolds in osteogenic media for 7 days prior to implantation resulted in increased defect closure from $3.43 \pm 1.22 \%$ to $13.7 \pm 7.31 \%$ from 4 to 12 weeks. The non-photoactivated PC-miR-148b-SNP conjugate closure percentage increased insignificantly from $3.83 \pm 1.19 \%$ to $5.46 \pm 2.01 \%$ ( $P>0.05$; one-sample $t$-test $)$ while the percentage closure values for photoactivated miR-148b conjugates increased significantly from $6.54 \pm 4.28 \%$ to $32.53 \pm 8.3 \%$ ( $P<0.05$; one-sample $t$-test) (Fig. $5 \mathrm{~A}$ and B).

In the samples that received Matrigel ${ }^{\mathrm{TM}}$, there was no significant difference in the closure of the negative control defect from the experimental group defects after 12 weeks. Defect closure was moderate in the Matrigel $^{\mathrm{TM}}$-only sample $(18.98 \%)$. Defect closure of Matrigel ${ }^{\mathrm{TM}}$ + PC-miR148b-SNP samples corresponds to $19.34+1.79 \%$, and defect closure of Matrigel $^{\mathrm{TM}}+$ PC-miR148b-SNP + Flashed samples corresponds to $17.7+1.34 \%$ (Fig. 5C).

\subsection{Histological analysis}

The mouse calvarium sections were decalcified with EDTA and the presence of collagen was studied with H\&E and Masson's Trichrome stains. The images in Fig. 6 track the changes in collagen lining across the defects treated with PC-miR148b-SNP conjugates in PCL scaffolds between 4 and 12 weeks. Comparing the images taken at 4 and 12 weeks, the pink-stained collagen lining remained consistently thin and minimal in the negative controls, scaffold only, empty defect samples and scaffold + hASCs (Fig. 6: 1a-3a and 1b-3b). For positive control, scaffold + hASCs $(\mathrm{OM})$, the collagen lining appeared visibly thicker and 
mineralized with purple-stained bone marrow present in the new bone (Fig. 6: 4b). The greatest difference was seen between the photo and non-photoactivated PC-miR-148b-SNP conjugate samples. In the non-photoactivated sample, few strands of collagen fiber lining stretched across the defect site between the pre-existing calvarium but mineralization is minimal (Fig. 6: 5a and 5b). For photoactivated PC-miR-148b-SNP conjugates, the collagen fiber lining can be seen thickening between 4 and 12 weeks (Fig. 6: 6a and 6b). The collagen lining thickened the most at 12 weeks and was of the same density and thicknesses as the native calvarium.

Similar trends were seen when the sections were stained with Masson's Trichrome. The intercellular fiber and collagen appear red and blue, respectively. The negative control samples—scaffold only and empty defect samples-demonstrated the absence of collagen at the defect site (Fig. 6: 1c and 2c). Small blue-stained collagen colonies were seen at the interface between the preexisting bone and the defect site. Scaffold + hASC samples show an increased presence of collagen at the interface but the lining did not span across the defect site. There was minimal closure/healing of the defect in the negative controls. The positive control group, scaffold + hASCs (OM), showed an increased ossification, greater than that of the native bone. After 12 weeks, the hASC scaffolds mineralized completely due to osteogenic induction prior to implantation. The mineralized and ossified scaffolds had similar staining to the calvarium (Fig. 6: 4c). Differences in the amount of collagen can be appreciated by comparing the stained sections of non-photoactivated and photoactivated PCmiR-148b-SNP conjugates (Fig. 6: 5c and 6c). The collagen strands thicken as the scaffolds mineralize with the photoactivation of conjugates.

The improved defect closure occurred in the animals implanted with scaffolds including photo-released miR-148b. These transverse sections of the new bone with photoactivated PC-miR-148b-SNP conjugate showed an abundant amount of collagen present at the defect site. Formation of the new bone was also appreciated in association with a few colonies of dark purple bone marrow present within the thick collagen lining.

\subsection{In vivo silver distribution}

A critical question surrounding the use of plasmonic particles in vivo is biotransformation, fate and toxicity. While histology of the CSD site did not indicate localized toxicity or tissue damage, no systemic toxicity was observed and no subject animals were lost during the study, the question still remained as to the fate of the PC-miR-148b-SNP conjugates and systemic distribution. To determine the final tissue distribution and terminal location of silver after 12 weeks, the concentration of silver in photoactivated samples was assessed in brain, liver, spleen and kidney, corresponding to $0.40 \pm 0.19,0.12 \pm 0.07,1.20 \pm 0.65$ and $0.26 \pm 0.06 \mathrm{ppm}$, respectively (Fig. 7). Previous research has shown that similarly - sized gold nanoparticles have also accumulated in kidneys, liver, spleen, brain and stomach [2123]. The concentrations of silver in all the measured organs, for both photoactivated and non-photoactivated samples, were not statistically significant from the non-treated control mice. We hypothesize that the low dose of PC-miR148b-SNP conjugate $\left(\sim 16 \mu \mathrm{g} \mathrm{g}^{-1}\right.$ mice $)$ used to initiate healing of the defect may explain the close proximity of the values to the control mice. 
All the animals appeared healthy with no visible signs of distress or loss of function. These results coincide with the previously reported in vitro results, which demonstrated no significant cytotoxicity of miR-148b-SNP conjugates with or without light activation when transfected in hASCs [11]. These results further support that the PC-miR-148b-SNP conjugates delivered at the effective concentration $(0.60 \mathrm{nM})$ used in this in vivo study have limited toxicity and an acceptable therapeutic index.

\section{Discussion}

Differentiation-inducing systems providing temporal control have the potential to improve outcomes in surgical reconstruction by precise modulation of wound healing and tissue repair processes. This photoactivated technique, particularly when combined with human autologous progenitor cells and miRNAs delivered in a nanoformulated vehicle, could result in a new regenerative medicine paradigm.

Modulation of gene expression with miRNA is a promising technique for improving control over differentiation. Recent efforts to modulate the osteogenic differentiation of hASCs have been conducted in vitro, demonstrating the upregulation of early and late stage osteogenic markers, alkaline phosphatase, osteocalcin, Runx2 and Alizarin Red staining when photoactivated [11]. In this in vivo study we analyzed the healing of the critical size defect in the mouse calvarium with a particle system for delivery of a truncated miR-148b mimic.

It was interesting to observe that in vitro calcification of hASCs was significantly higher in Matrigel than in PCL scaffolds. Matrigel is a gelatinous protein that resembles the complex extracellular environment found in many tissues and its heterogenous composition consists of various proteins and growth factors. The close resemblance of Matrigel to ECM enhances its ability to house hASCs and become differentiated with photoactivated PC-miR148b-SNP conjugates.

The PC-miR-148b-SNP photoactivated conjugate was evaluated here in a well-described critical size defect model [16] and shown to have promising results. PCL was chosen as a scaffold for this study as it has previously been shown to have little impact on hASC differentiation, providing a convenient method to isolate the osteogenic activity of the PCmiR-148b-SNP photoactivated conjugate without osteogenic substrate effects [24]. The defect partially healed with the photoactivated conjugates while the control groups had minimal healing and remained opened even after 12 weeks, further verifying the model and the size of the non-healing defect. A progression was observed in the percentage closure of the defect between 4 and 12 weeks in all the samples.

Interestingly, improved mineralization results with Matrigel in vitro do not translate into in vivo CSD healing, where we observed non-significant closure of the defect with the photoactivated PC-miR148b-SNP conjugates. It is hypothesized that these results may be related to mechanical properties or organization of the hydrogel as previous studies have indicated a correlation between substrate rigidity and osteogenic differentiation in progenitor cells [25-28]. Trappmann et al. conclude that the osteogenic differentiation of MSCs is influenced by the stiffness of polyacrylamide, but that the driver for cell adhesion and differentiation is actually ECM organization, which is itself dictated by the mechanical 
properties and morphology of the underlying substrate [29]. Therefore, a possible explanation for the incongruence of the in vitro and in vivo results may be variation in the Matrigel organization as a function of substrate. As an alternative it is also possible that in vivo outcomes may be a result of Matrigel degradation by proteases post implantation. In contrast to PCL scaffolds, the Matrigel may not be chemically and physically stable in the CSD environment as a result of protease activity, and therefore is unable to promote longterm osteogenic differentiation and bone formation. These results indicate that scaffold composition could play an important role when directing stem cell differentiation via silencing nucleic acid techniques in vivo.

The histological analysis on the decalcified calvarium sections also demonstrated the thickening of the collagen fiber lining, across the defect, when the photoactivated conjugates were used. The collagen stained with HE and Masson's Trichrome displayed the same degree of thickness as present in the native calvarium and the new bone was infused with older bone in the photoactivated conjugates samples after 12 weeks.

Several successful attempts have been made in vitro to induce miRNA-mediated osteoblast differentiation from osteoprogenitor cells [11,30-33] but the in vivo and clinical translation of these results have been very limited. Only a handful of in vivo studies have been conducted to differentiate stem cells, from various sources, to osteoblasts leading to the formation of bone and closure of a critical sized defect. The PCL percentage closure values, in this study, are very competitive and comparable to the other limited studies using similar miRNA/stem cell-modulated approaches to improve bone formation. Deng et al. showed that scaffolds implanted with rat-derived bone marrow and adipose-derived stem cells transfected with antisense miR-31 resulted in $\sim 35 \%$ closure of the calvarial defect $[34,35]$. Using a rodent subcutaneous model to evaluate the in vivo differentiation of stem cells, Chen et al. demonstrated that anti-miR-34a improved bone formation 10\% [36]. Combination approaches using hASC treated with BMP-2 in conjunction with a hydroxyapatite containing PLGA scaffold resulted in more complete defect closure but it is expected that with further refinement and optimization of the scaffold composition, hASC concentration and miRNA mimic dose that comparable closure rates can be achieved using this photomodulated nucleic acid-based strategy [16]. Compared to previous miRNA mimic-, siRNA- or BMP-based strategies for inducing osteogenesis in vivo, this technique has a potential advantage in that it provides for spatiotemporal control of induction and can be adapted to a myriad of oligonucleotide sequences. We believe this technology holds great promise as not only can the hASCs be conveniently delivered to the defect site, but for the first time stem cell differentiation can be controlled temporally, in vivo, via photon exposure. Future applications might allow similar use and activation in a chronic or nonhealing bone fracture.

\section{Conclusions}

This study demonstrates that nanoformulated miRNA-based conjugates released in scaffolds via photoactivation can lead to the induced differentiation of hASCs in an in vivo model of bone healing. The photo-osteoblasts in the PCL scaffold healed the CSD by $32.53 \pm 8.3 \%$. While the results of this study demonstrate the ability of the photoactivated PC-miR-148b- 
SNP conjugate to effectively modulate hASC osteogenic differentiation and drive significantly improved CSD closure, translating this technology into a clinical setting will likely require better matrices or scaffolding materials permitting improved light penetration and cell distribution. Future efforts may potentially explore thermogelating hydrogels and fibrin/thrombin matrices as potential injectable and scaffolds with high optical penetration depths.

\section{Acknowledgments}

We thank NanoHorizons Inc. (Bellefonte, PA) for providing us with silver nanoparticles. Financial support from the National Science Foundation grants (Proposal numbers CMMI-963482 and CBET-1254281) and from LSU AgCenter is acknowledged. B.L. is funded by NIH-1K08GM109105-01. We also thank Mollie Smoak from the LSU Biological Engineering program with assisting in processing samples for ICP-OES.

\section{References}

1. Fang TD, Nacamuli RP, Song HJM, Fong KD, Shi Y-Y, Longaker MT. Guided tissue regeneration enhances bone formation in a rat model of failed osteogenesis. Plast Reconstr Surg. 2006; 117:1177-85. [PubMed: 16582784]

2. Gimble J, Guilak F. Adipose-derived adult stem cells: isolation, characterization, and differentiation potential. Cytotherapy. 2003; 5:362-9. [PubMed: 14578098]

3. Zuk PA, Zhu M, Ashjian P, De Ugarte DA, Huang JI, Mizuno H, et al. Human adipose tissue is a source of multipotent stem cells. Mol Biol Cell. 2002; 13:4279-95. [PubMed: 12475952]

4. Dragoo JL, Lieberman JR, Lee RS, Deugarte DA, Lee Y, Zuk PA, et al. Tissue-engineered bone from BMP-2 to transduced stem cells derived from human fat. Plast Reconstr Surg. 2005; 115:1665-73. [PubMed: 15861072]

5. Gimble JM, Katz AJ, Bunnell BA. Adipose-derived stem cells for regenerative medicine. Circ Res. 2007; 100:1249-60. [PubMed: 17495232]

6. Zuk PA, Zhu M, Mizuno H, Huang J, Futrell JW, Katz AJ, et al. Multilineage cells from human adipose tissue: implications for cell-based therapies. Tissue Eng. 2001; 7:211-28. [PubMed: 11304456]

7. Schoolmeesters A, Eklund T, Leake D, Vermeulen A, Smith Q, Aldred SF, et al. Functional profiling reveals critical role for miRNA in differentiation of human mesenchymal stem cells. PLoS ONE. 2009; 4:9.

8. Luzi E, Marini F, Sala SC, Tognarini I, Galli G, Brandi ML. Osteogenic differentiation of human adipose tissue-derived stem cells is modulated by the miR-26a targeting of the SMAD1 transcription factor. J Bone Miner Res. 2008; 23:287-95. [PubMed: 18197755]

9. Mizuno Y, Yagi K, Tokuzawa Y, Kanesaki-Yatsuka Y, Suda T, Katagiri T, et al. MiR-125b inhibits osteoblastic differentiation by down-regulation of cell proliferation. Biochem Biophys Res Commun. 2008; 368:267-72. [PubMed: 18230348]

10. Luzi E, Marini F, Sala SC, Tognarini I, Galli G, Brandi ML. Osteogenic differentiation of human adipose tissue-derived stem cells is modulated by the miR-26a targeting of the SMAD1 transcription factor. J Bone Miner Res J Am Soc Bone Miner Res. 2008; 23:287-95.

11. Qureshi AT, Monroe WT, Dasa V, Gimble JM, Hayes DJ. MiR-148b-Nanoparticle conjugates for light mediated osteogenesis of human adipose stromal/stem cells. Biomaterials. 2013

12. Mendes S, Tibbe J, Veenhof M, Bakker K, Both S, Platenburg P, et al. Bone tissue-engineered implants using human bone marrow stromal cells: effect of culture conditions and donor age. Tissue Eng. 2002; 8:911-20. [PubMed: 12542937]

13. Harris S, Bonewald L, Harris M, Sabatini M, Dallas S, Feng J, et al. Effects of transforming growth factor $\beta$ on bone nodule formation and expression of bone morphogenetic protein 2 , osteocalcin, osteopontin, alkaline phosphatase, and type I collagen mRNA in long-term cultures of fetal rat calvarial osteoblasts. J Bone Miner Res. 2009; 9:855-63. [PubMed: 8079661] 
14. Shea LD, Wang D, Franceschi RT, Mooney DJ. Engineered bone development from a preosteoblast cell line on three-dimensional scaffolds. Tissue Eng. 2000; 6:605-17. [PubMed: 11103082]

15. Wan DC, Pomerantz JH, Brunet LJ, Kim JB, Chou YF, Wu BM, et al. Noggin suppression enhances in vitro osteogenesis and accelerates in vivo bone formation. J Biol Chem. 2007; 282:26450-9. [PubMed: 17609215]

16. Levi B, James AW, Nelson ER, Vistnes D, Wu B, Lee M, et al. Human adipose derived stromal cells heal critical size mouse calvarial defects. PLoS ONE. 2010; 5:e11177. [PubMed: 20567510]

17. Levi B, Nelson ER, Li S, James AW, Hyun JS, Montoro DT, et al. Dura mater stimulates human adipose-derived stromal cells to undergo bone formation in mouse calvarial defects. Stem Cells. 2011; 29:1241-55. [PubMed: 21656608]

18. Qureshi AT, Terrell L, Monroe WT, Dasa V, Janes ME, Gimble JM, et al. Antimicrobial biocompatible bioscaffolds for orthopaedic implants. J Tissue Eng Regen Med. 2012

19. Cowan CM, Shi Y-Y, Aalami OO, Chou Y-F, Mari C, Thomas R, et al. Adipose-derived adult stromal cells heal critical-size mouse calvarial defects. Nat Biotechnol. 2004; 22:560-7. [PubMed: 15077117]

20. Forman J, Dietrich M, Monroe WT. Photobiological and thermal effects of photoactivating UVA light doses on cell cultures. Photochem Photobiol Sci. 2007; 6:649-58. [PubMed: 17549267]

21. Chen Y-S, Hung Y-C, Liau I, Huang GS. Assessment of the in vivo toxicity of gold nanoparticles. Nanoscale Res Lett. 2009; 4:858-64. [PubMed: 20596373]

22. Sonavane G, Tomoda K, Makino K. Biodistribution of colloidal gold nanoparticles after intravenous administration: effect of particle size. Colloids Surf, B. 2008; 66:274-80.

23. De Jong WH, Hagens WI, Krystek P, Burger MC, Sips AJ, Geertsma RE. Particle size-dependent organ distribution of gold nanoparticles after intravenous administration. Biomaterials. 2008; 29:1912-9. [PubMed: 18242692]

24. Zanetti A, McCandless G, Chan J, Gimble J, Hayes D. Characterization of novel akermanite: poly$\varepsilon$-caprolactone scaffolds for human adipose-derived stem cells bone tissue engineering. J Tissue Eng Regen Med. 2012

25. Chan B, Leong K. Scaffolding in tissue engineering: general approaches and tissue-specific considerations. Eur Spine J. 2008; 17:467-79. [PubMed: 19005702]

26. Rowlands AS, George PA, Cooper-White JJ. Directing osteogenic and myogenic differentiation of MSCs: interplay of stiffness and adhesive ligand presentation. Am J Physiol Cell Physiol. 2008; 295:C1037-44. [PubMed: 18753317]

27. Shih YRV, Tseng KF, Lai HY, Lin CH, Lee OK. Matrix stiffness regulation of integrin-mediated mechanotransduction during osteogenic differentiation of human mesenchymal stem cells. J Bone Miner Res. 2011; 26:730-8. [PubMed: 20939067]

28. Pek YS, Wan AC, Ying JY. The effect of matrix stiffness on mesenchymal stem cell differentiation in a 3D thixotropic gel. Biomaterials. 2010; 31:385-91. [PubMed: 19811817]

29. Trappmann B, Gautrot JE, Connelly JT, Strange DG, Li Y, Oyen ML, et al. Extracellular-matrix tethering regulates stem-cell fate. Nat Mater. 2012; 11:642-9. [PubMed: 22635042]

30. Kapinas K, Kessler CB, Delany AM. MiR-29 suppression of osteonectin in osteoblasts: regulation during differentiation and by canonical Wnt signaling. J Cell Biochem. 2009; 108:216-24. [PubMed: 19565563]

31. Zhang J, Fu W, He M, Xie W, Lv Q, Wan G, et al. MiRNA-20a promotes osteogenic differentiation of human mesenchymal stem cells by co-regulating BMP signaling. RNA Biol. 2011; 8:829-38. [PubMed: 21743293]

32. Kim YJ, Bae SW, Yu SS, Bae YC, Jung JS. MiR-196a regulates proliferation and osteogenic differentiation in mesenchymal stem cells derived from human adipose tissue. J Bone Miner Res. 2009; 24:816-25. [PubMed: 19063684]

33. Mizuno Y, Tokuzawa Y, Ninomiya Y, Yagi K, Yatsuka-Kanesaki Y, Suda T, et al. MiR-210 promotes osteoblastic differentiation through inhibition of AcvRlb. FEBS Lett. 2009; 583:2263-8. [PubMed: 19520079] 
34. Deng Y, Bi X, Zhou H, You Z, Wang Y, Gu P, et al. Repair of critical-sized bone defects with anti-miR-31-expressing bone marrow stromal stem cells and poly (glycerol sebacate) scaffolds. Eur Cells Mater. 2014; 27:13.

35. Deng Y, Zhou H, Zou D, Xie Q, Bi X, Gu P, et al. The role of miR-31-modified adipose tissuederived stem cells in repairing rat critical-sized calvarial defects. Biomaterials. 2013; 34:6717-28. [PubMed: 23768901]

36. Chen L, Holmstrøm K, Qiu W, Ditzel N, Shi K, Hokland LB, et al. MicroRNA-34a Inhibits osteoblast differentiation and in vivo bone formation of human stromal stem cells. Stem Cells. 2013

\section{Appendix A. Figures with essential colour discrimination}

Certain figures in this article, particularly Figures 2 and 6, are difficult to interpret in black and white. The full colour images can be found in the on-line version, at http://dx.doi.org/ 10.1016/j.actbio.2014.10.010. 
(A)

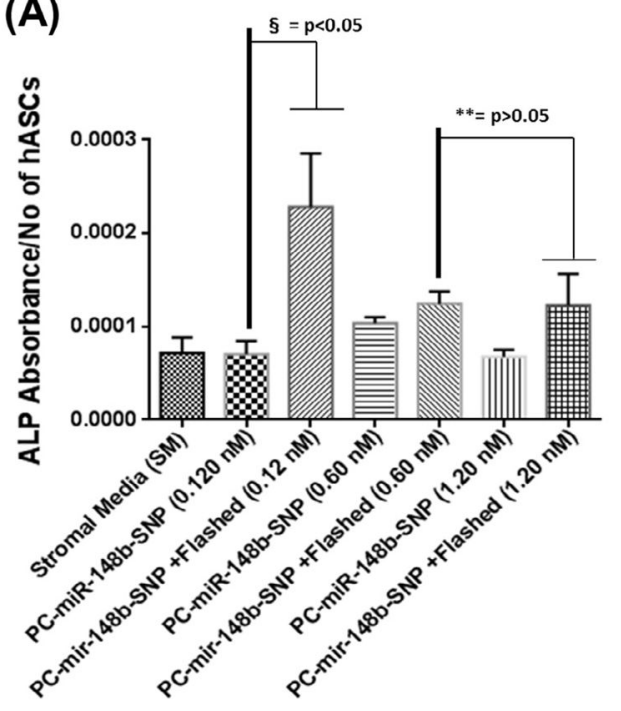

(B)

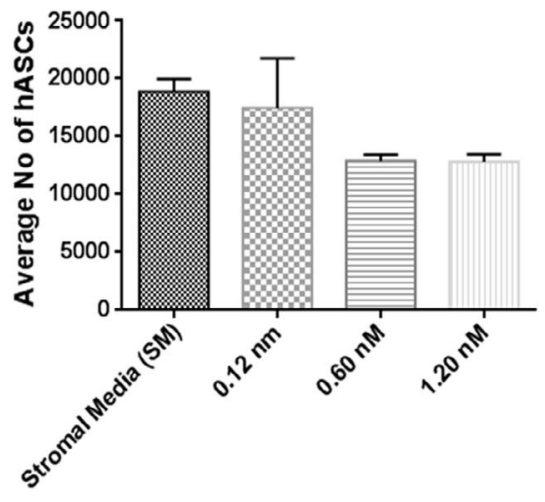

Fig. 1.

(A) Quantification of ALP absorbance after 7 days for various concentrations of PCmiR-148b conjugates. (B) Average number of hASCs after 7 days. $\S$ denotes significantly different values $(P<0.05$; one-sample $t$-test $)$ while $* *$ denotes insignificantly different values $(P>0.05$; one-sample $t$-test $)$. 

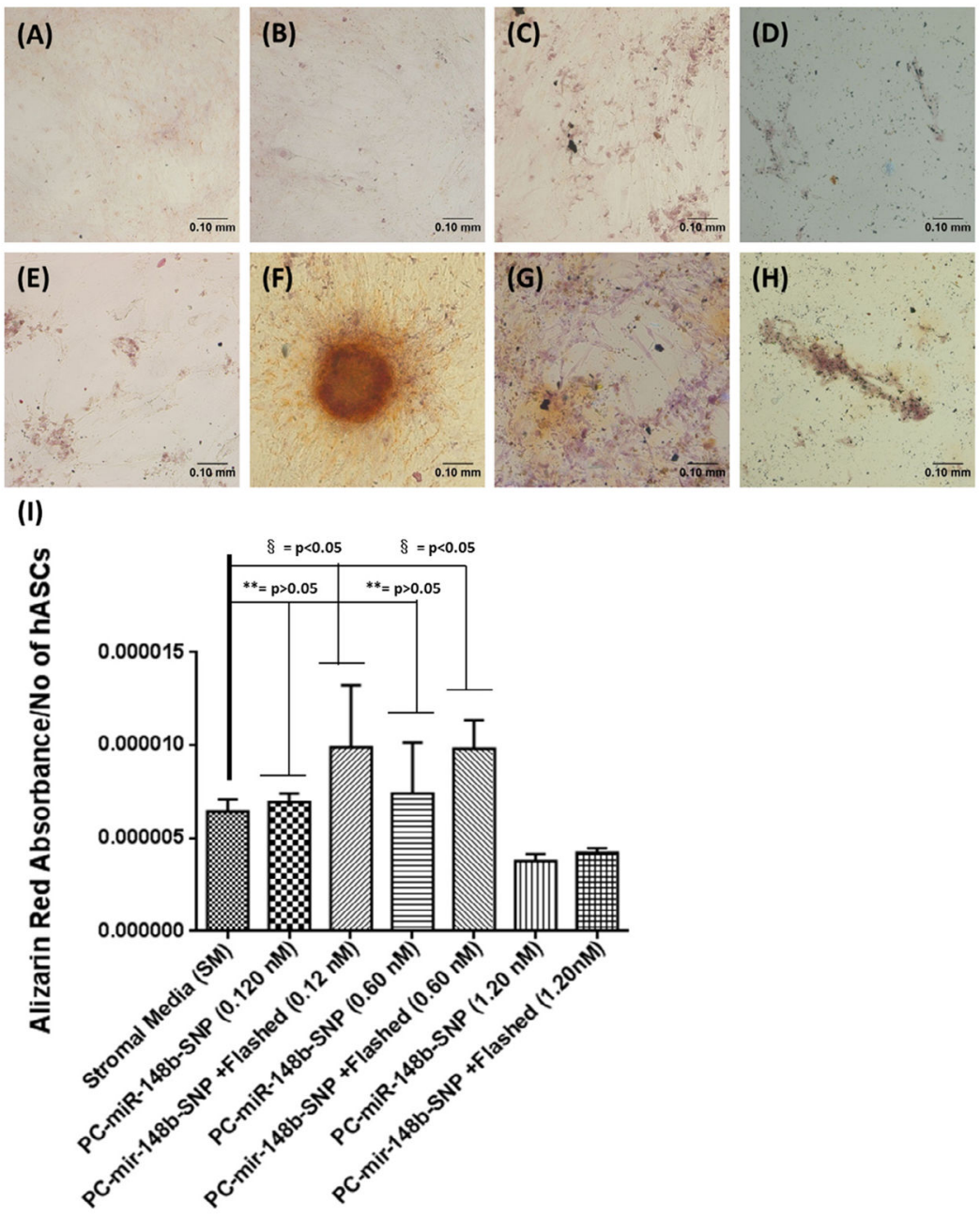

Fig. 2.

Alizarin Red stained hASCs in (A) stromal media, (B) 0.12 nM PC-miR-148b-SNP, (C) $0.60 \mathrm{nM}$ PC-miR-148b-SNP, (D) $1.20 \mathrm{nM}$ PC-miR-148b-SNP, (E) hASCs + Flashed, (F) $0.12 \mathrm{nM}$ PC-miR-148b-SNP + Flashed, (G) $0.60 \mathrm{nM}$ PC-miR-148b-SNP + Flashed and (H) $1.20 \mathrm{nM}$ PC-miR-148b-SNP + Flashed. (I) Quantification of Alizarin Red absorbance after 14 days across varying concentration of PC-miR-148b conjugates. $§$ denotes significantly different values $(P<0.05$; one-sample $t$-test) while $* *$ denotes insignificantly different values $(P>0.05$; one-sample $t$-test $)$. 


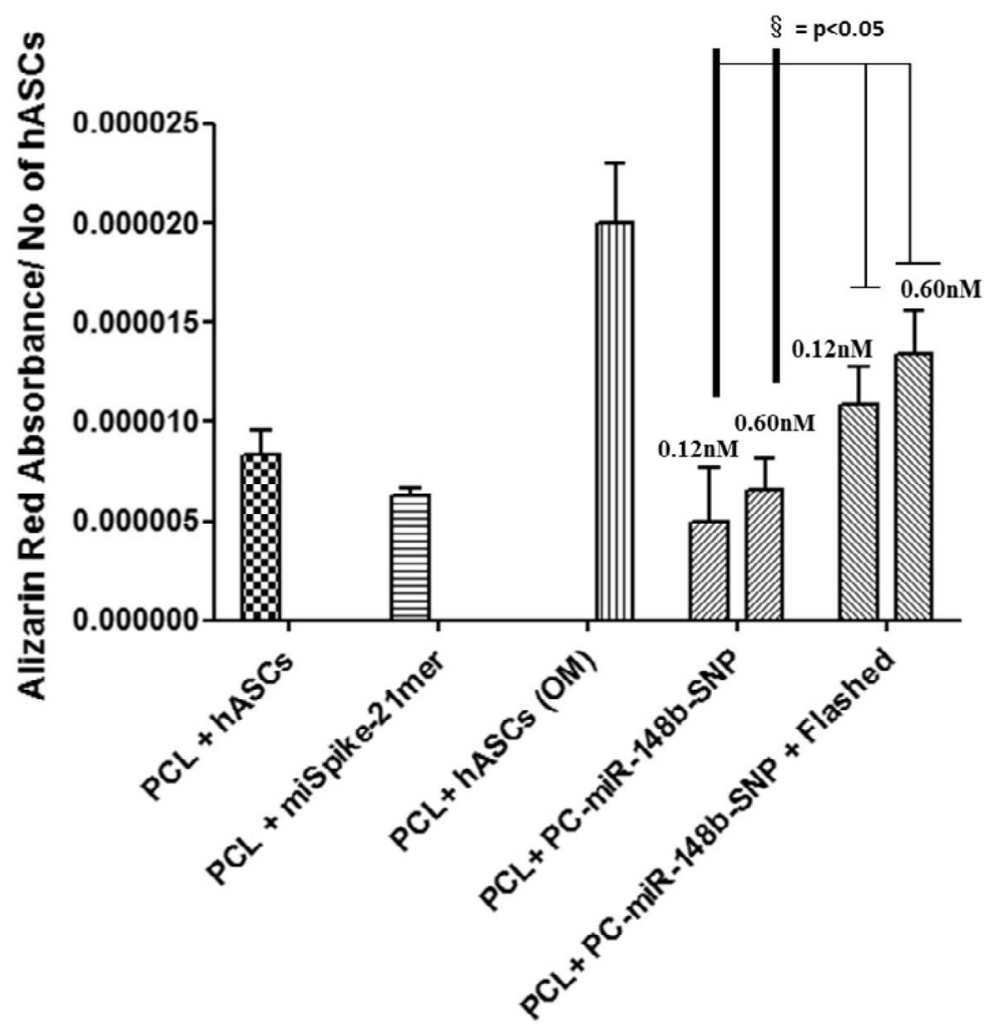

Fig. 3.

Ex vivo normalized Alizarin Red absorbance of PCL scaffolds loaded with hASCs and various concentrations of PC-miR-148-SNP conjugates $(0.12$ and $0.60 \mathrm{nM})$. $\S$ denotes significantly different values $(P<0.05$; one-sample $t$-test). 


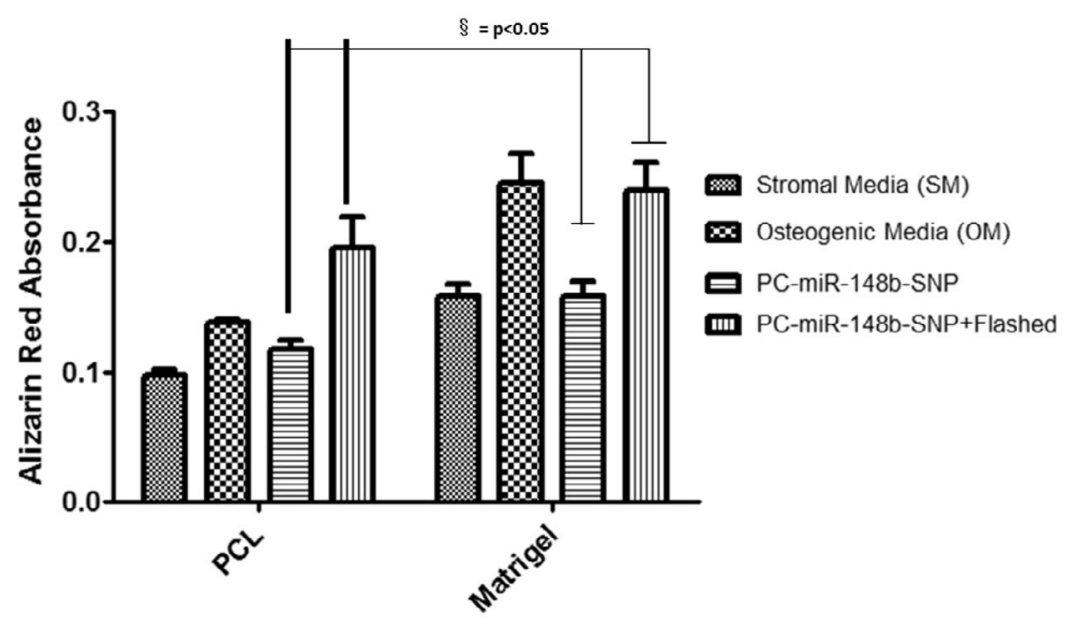

Fig. 4.

In vitro Alizarin Red stain uptake comparing of $\mathrm{PCL}$ and Matrigel ${ }^{\mathrm{TM}}$ scaffolds loaded with hASCs and PC-miR148-SNP conjugates. $§$ denotes significantly different values $(P<0.05$; one-sample $t$-test). 


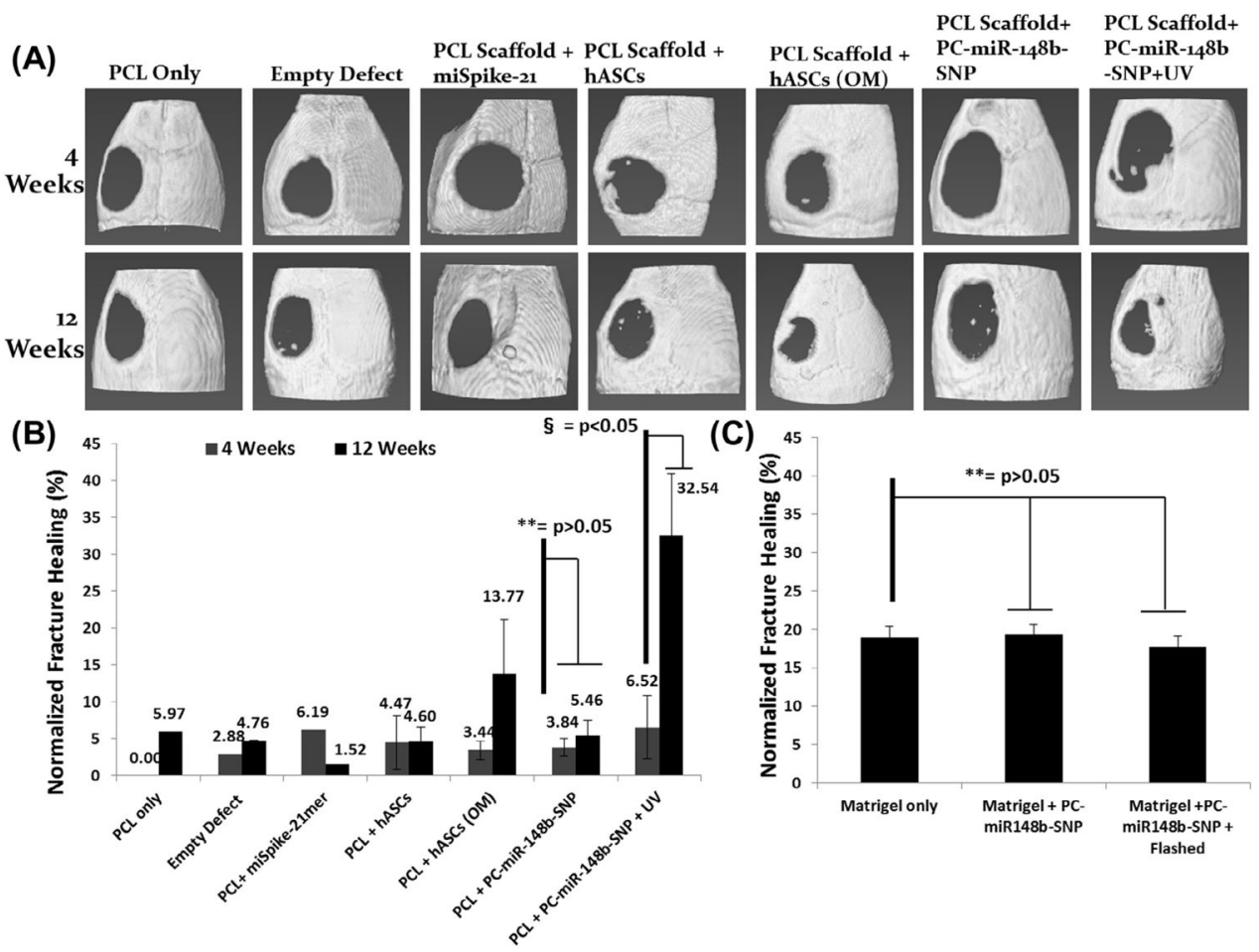

Fig. 5.

(A) 3-D reconstructed images of $4 \mathrm{~mm}$ calvarial size defects. The defect was imaged with $\mu \mathrm{CT}$ after killing the mice 4 and 12 weeks post implantation of the PCL scaffold with photo and non-photoactivated PC-miR-148b-SNP conjugates. The percentage healing/closure of the defect were normalized to the animal's preexisting bone for (B) PCL scaffolds and (C) Matrigel scaffolds. § denotes significantly different values $(P<0.05$; one-sample $t$-test $)$ while ** denotes insignificantly different values $(P>0.05$; one-sample $t$-test $)$. 

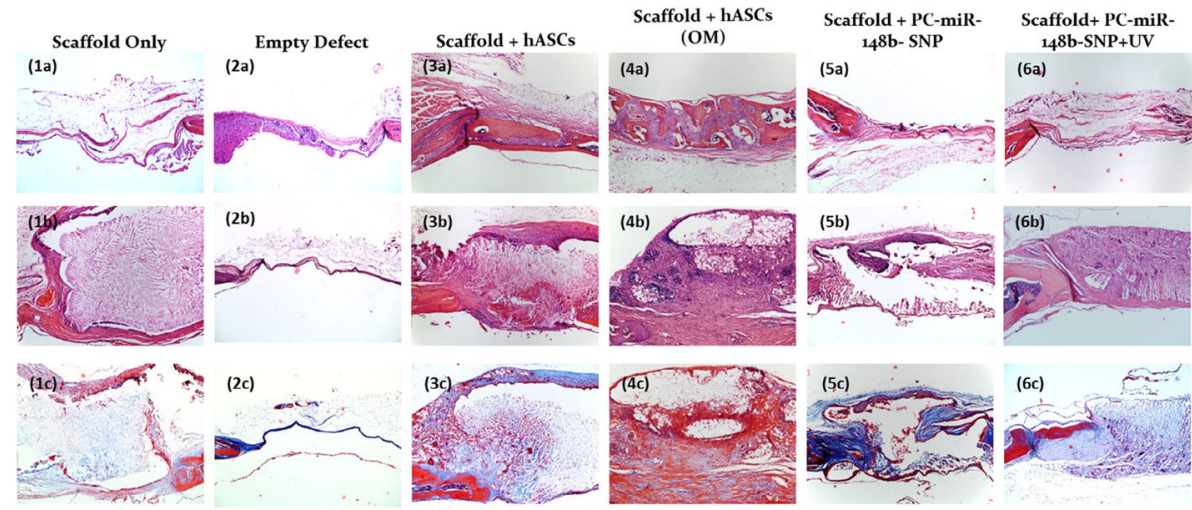

Fig. 6.

The mouse calvaria were decalcified, paraffin-embedded, sectioned and stained with (a) HE stain/4 weeks, (b) HE stain/12 weeks and (c) Masson's Trichrome/12 weeks to study the presence of collagen. The collagen appears pink and blue with HE and Trichrome stain, respectively. The images were taken with Olympus BX46 and magnification is 10x. 


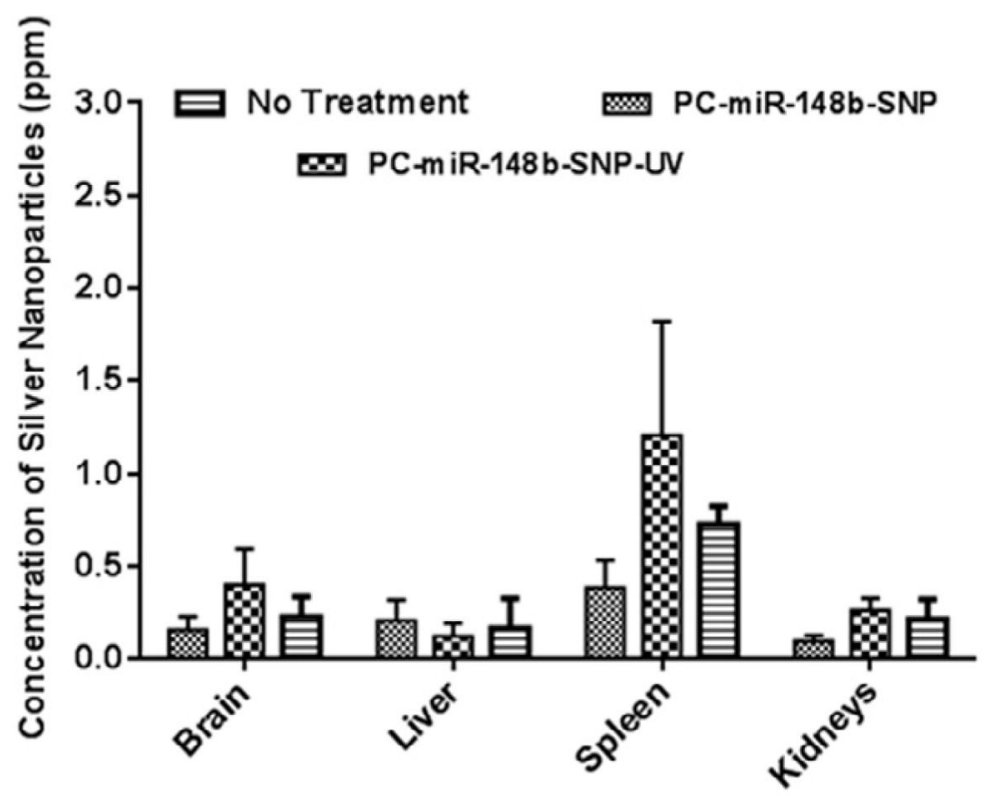

Fig. 7.

Biodistribution of silver in different organs of mice after 12 weeks. The concentration values are not statistically different from those of non-treated mice. 